\title{
Development and Evaluation of Loop-Mediated Isothermal Amplification (LAMP) Assay for Quick Identification of Three Japanese Toads
}

\author{
Koshiro ETO $^{1 *}{ }^{*}$, MASAFUmi MATSUI ${ }^{1}$, KANTO NISHIKAWA $^{1}$, \\ AND TAKASHI HARAMURA ${ }^{2}$ \\ ${ }^{1}$ Graduate School of Human and Environmental Studies, Kyoto University, Yoshida \\ Nihonmatsu-cho, Sakyo-ku, Kyoto 606-8501, JAPAN \\ ${ }^{2}$ Field Science Education and Research Center, Kyoto University, 459 Shirahama, \\ Nishimuro, Wakayama 649-2211, JAPAN
}

\begin{abstract}
Three species/subspecies of toads (Bufo japonicus japonicus, $B . j$. formosus, and $B$. torrenticola) show parapatric/sympatric patterns of distribution in Japan, and usually they are morphologically indistinguishable at early developmental stages. For quick and easy genetic identification of these three toads, we validated LAMP assay. We designed taxon-specific primers for LAMP assay on mitochondrial cytochrome $b$ region, and these primer sets accurately assigned DNA samples derived from three toads to each taxon. Due to its instancy and simplicity, LAMP method could be a useful diagnostic tool to distinguish taxa which resemble each other.
\end{abstract}

Key words: LAMP; Bufo; Cytochrome b; Species identification

\section{INTRODUCTION}

Because molecular techniques have come into wide use, now it is becoming common to employ genetic methods such as DNA barcoding to identify taxa and intraspecific lineages of various organisms. However, DNA sequencing is still costly to conduct, and it is sometimes unsuitable in cases where many samples need to be analyzed. Therefore several alternative methods (e.g., PCR-RFLP: Okamoto and Hikida, 2009; Igawa et al., 2015) have been developed to make genetic identification easier, but most of them require special equipment such as thermal cycler to

* Corresponding author. Tel: +81 75-753-2890; FAX: +81 75-753-2891;

E-mail address: koshiro.eto@gmail.com run experiments.

Loop-mediated Isothermal Amplification (LAMP) is a unique technique to amplify fragments of DNA under a constant temperature using four or six primers (Notami et al., 2000). Using fluorescent detection reagent and Blue LED/UV rays, it can detect specific sequences simply by eye. Due to its simplicity, LAMP method is recently used to detect pathogens (e.g., Aonuma et al., 2008), and some commercially available detection kits have been developed (Mori and Notami, 2009). This method is also used for species identification of parasites (Ai et al., 2010) and would also be applicable to other taxa.

To evaluate the availability of LAMP assay for species identification of amphibians, we preliminarily applied this method to distinguish three Japanese toads: Bufo japonicus 
japonicus, B. j. formosus, and B. torrenticola. The former two subspecies are still-water breeders and show parapatric pattern of distribution on Honshu Island, the main island of Japan (Matsui, 1984; Maeda and Matsui, 1999). In some areas of Central Honshu, B. j. formosus occurs sympatrically with $B$. torrenticola, which breeds in lotic environments and is reproductively isolated from B. japonicus subspecies (Matsui, 1976). These three toads can be identified by adult morphology, but they resemble each other at immature stages and are difficult to be differentiated. Larvae of $B . j$. japonicus and $B . j$. formosus are also indistinguishable, although $B$. torrenticola has larval traits distinct from B. japonicas subspecies (Matsui, 1976). The ability to distinguish these three toads in their early stages would be a basic step in conducting various studies of these species, such as on their taxonomy, ethology, and ecology. Moreover, it would also have important implications for conservation management of them. Using mitochondrial cytochrome b (cytb) sequence of the three toads, we designed taxon-specific primers for LAMP method to separate these taxa, and evaluated its accuracy and cost-effectiveness.

\section{Materials ANd Methods}

\section{Designing LAMP primers}

We used published cytb sequences of $B . j$. japonicas $(\mathrm{n}=6), B . j$. formosus $(16)$, and $B$. torrenticola (2) from Honshu and Shikoku islands (AB159232-AB159255, Igawa et al., 2006) to design primers. Comparing these sequences, we detected single nucleotide polymorphism (SNP) sites representing taxonspecific difference by eye as candidate target regions. Then the candidate LAMP primer sets specific to each region were designed with assistance of online software PrimerExplorer V4 (http://primerexplorer.jp/intro/index.html). We put the SNPs on the 3' end of at least one primer of each primer set (consisting of four primers: forward-inner [FI], forward-3 [F3], backward-inner [BI], and backward-3 [B3]). After examination with DNA samples derived from three toads (see below), a total of 10 primers (three sets) listed in Table 1 were chosen as the standard primer sets. All primers were prepared as $2 \mu \mathrm{M}$ with distilled water.

\section{Validation of LAMP assay for three toads}

To validate the availability and accuracy of designed LAMP primers, we extracted DNA of three species of Japanese toads from several different localities in Honshu: three B. j. japonicus from Shiga Prefecture (stored

TABLE 1. Primer sets designated for LAMP assay in this study.

\begin{tabular}{|c|c|c|}
\hline Target taxon & Primer codes & Sequences $\left(5^{\prime}=>3^{\prime}\right)$ \\
\hline \multicolumn{3}{|c|}{ Bufo japonicus japonicus } \\
\hline & FI_cytb_Bj & TACTGAAAATCCGCCTCAGATTCTCCGCCGCCCCCTAC \\
\hline & BI_cytb_Bj & AGTAGACAACGCAACTCTAACGTATAGAGGCGCCTGCAATG \\
\hline & F3_cytb_Bjf & GGGCAACCGTAATCACAAAC \\
\hline & B3_cytb_Bjf & GGGTTGGAGGATCCTGTYTG \\
\hline \multicolumn{3}{|c|}{ B. j. formosus } \\
\hline & FI_cytb_Bf & GACCGAAAAGCCGCCTCAGATTCTCTGCCGCCCCCTA \\
\hline & BI_cytb_Bf & GGTCGACAACGCAACTCTAACGTATAGAGGCGCCTGCAATG \\
\hline & F3_cytb_Bjf & GGGCAACCGTAATCACAAAC \\
\hline & B3_cytb_Bjf & GGGTTGGAGGATCCTGTYTG \\
\hline \multicolumn{3}{|c|}{ B. torrenticola } \\
\hline & FI_cytb_Bt & AGGATTAAGGCCTGTTGGGTGCAGGCGCCTCTATACACC \\
\hline & BI_cytb_Bt & TCСТАACTTCGACAAAATCCGGCCAGCAAAGCMAGTATG \\
\hline & F3_cytb_Bt & TTCACATTTCACTTCATCCT \\
\hline & B3_cytb_Bt & TTGTCTGGGTCACCTAGGA \\
\hline
\end{tabular}


at the Graduate School of Human and Environmental Studies, Kyoto University [KUHE], voucher no. 45512), Tottori Pref. (KUHE 49353), Ehime Pref. (KUHE 45506), and an unnumbered froglet from Okayama Pref.; four B. j. formosus from Aomori Pref. (KUHE 46299), Chiba Pref. (KUHE 46176), Shiga Pref. (KUHE 45512), and an unnumbered tadpole from Tochigi Pref.; and two $B$. torrenticola from Mie Pref. and Ishikawa Pref. (KUHE 3909 and 56072, respectively). Each DNA sample was extracted from frozen or ethanol-preserved tissue by using standard phenol-chloroform procedures or DNeasy Blood and Tissue Kit (QIAGEN) to compare the influences of the two different DNA extraction methods. The LAMP reaction was carried out with $10 \mu \mathrm{L}$ volume of reaction solution containing $1.0 \mu \mathrm{L}$ of template DNA solution, $0.8 \mu \mathrm{L}$ each of $\mathrm{FI}$ and $\mathrm{BI}$ primers, $0.1 \mu \mathrm{L}$ each of $\mathrm{F} 3$ and $\mathrm{B} 3$ primers, $0.4 \mu \mathrm{L}$ of Bst DNA Polymerase and $5.0 \mu \mathrm{L}$ of Reaction Mix (RM) of Loopamp DNA Amplification Kit (Eiken Chemical), and 0.4 $\mu$ L of Loopamp Fluorescent Detection (FD) Reagent (Eiken Chemical). The reaction mixtures were incubated at $63-65^{\circ} \mathrm{C}$ for $60-90 \mathrm{~min}$ and then heated at $95^{\circ} \mathrm{C}$ for $2 \mathrm{~min}$ to terminate the reaction. The reactions were performed by PCR thermal cycler Dice (TaKaRa) or dry thermo unit DTU-neo (Taitec). We accordingly added positive and negative controls to check cross-contamination. After reaction has finished, the reaction tubes were examined under commercially available blue LED tape for interior. The fluorescence intensity was checked through an orange acrylic plate. To evaluate the result of each tube, we compared the fluorescence intensity of reaction tubes with that of negative controls (template solution was replaced by distilled water) by eye. If the target SNPs existed in reaction solution, primers match these regions and LAMP reaction proceeds, and the byproduct of amplification reacts with FD, then the solution fluoresces strongly. To confirm that the target regions are surely amplified, we conducted standard gel electrophoresis for some samples under the following conditions: using $2 \% \mathrm{w} / \mathrm{v}$ Agarose S (Nippon Gene) gel with Midori Green Advance (Nippon Genetics), samples were run with TAE buffer for V100/15 min and then viewed under UV light with transilluminator NTM-10 (Funakoshi).

\section{RESUlts}

Positive and negative patterns of tube fluorescence and gel electrophoresis are shown in Fig. 1. There is no difference between the results of reaction performed in thermal cycler and dry thermo unit, although it seems that there was the difference of amplification efficiency among primer sets. The primer set for $B$. $j$. japonicus has better amplification efficiency than the other two sets: e.g., the incubation time of $60 \mathrm{~min}$ is enough for $B$. j. japonicus primer set, whereas the other two sets usually require $90 \mathrm{~min}$. However, in this study we did not adjust all of the template concentration as equal, so detail about the efficiency difference was unclear.

In the tube fluorescence method, each of LAMP products for the matched primers-
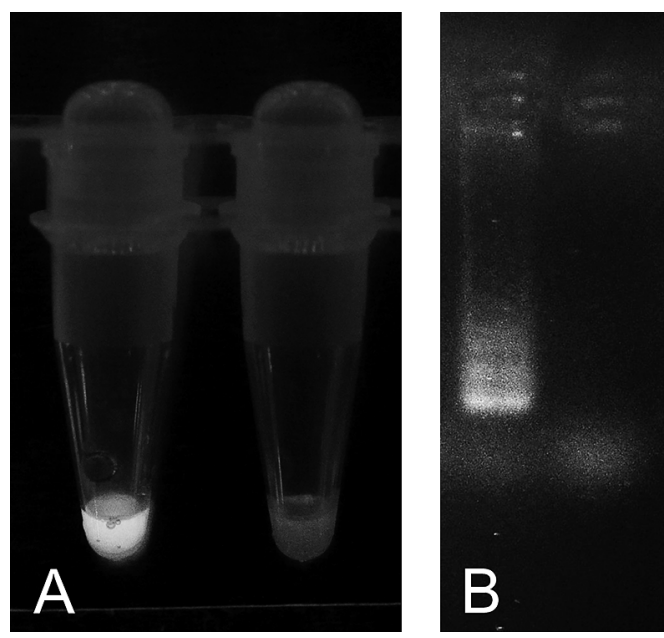

FIG. 1. A result of LAMP reaction with matched(left: genomic DNA of $B . j$. japonicus with primer set for $B . j$. japonicus) and mismatched- (right: genomic DNA of $B . j$. formosus with primer set for B. j. japonicus) primers-taxon combinations. (A) Detection by fluorescence reagent with blue LED. (B) Detection by gel electrophoresis. 
taxon combinations fluoresced stronger than the mismatched combinations and negative control. However, the mismatched/negative tubes also fluoresced weakly, and it was necessary to compare the relative strength with positive/negative controls to judge the results. Especially, DNA templates dissolved in Tris-EDTA (TE) buffer (samples extracted by phenol-chloroform procedures) sometimes fluoresced stronger than templates in elution buffer (buffer AE) of the DNeasy kit. It would be because TE buffer contains higher concentration of EDTA $(1.0 \mathrm{mM})$ than AE buffer $(0.5 \mathrm{mM})$. Chelators such as EDTA could react with FD reagent without the template DNA (Eiken genome site: http://www.eiken. co.jp/), so different concentrations of chelator would affect the strength of background fluorescence. Thus, different buffer should not be used in the same system, although the mismatched combinations with TE buffer fluoresced even weaker than the matched combinations with AE buffer.

In the gel electrophoresis, a typical band pattern of LAMP reaction was observed in the matched primers-taxon combinations. In LAMP, usually the bands show smear-like patterns because various length of DNA fragments are produced through the reaction. The gel electrophoresis is more accurate than the fluorescence method, as with the latter we have to judge the result by relative fluorescence intensity and it may sometimes cause decision error because of the range of of background strength. Thus, at least until the condition setting of experiment system is completed, it would be better to use both the gel electrophoresis and the fluorescence method to judge the result of LAMP reaction.

\section{Discussion}

Compared with other assays such as DNA barcoding and PCR-RFLP, LAMP is much more instant in experimental protocols. DNA barcoding protocol requires six steps: amplification of target DNA by PCR, purification of products, amplification and fluorescent labeling by CSR, re-purification, sequencing, and analysis of sequences. PCR-RFLP protocol is much simpler than barcoding and consists of three steps; amplification, incubation with restriction enzyme, and gel electrophoresis. However, LAMP protocol is even simpler, containing only two steps; amplification and gel electrophoresis. Furthermore, if fluorescence method is applicable, gel electrophoresis step can be omitted.

From the viewpoint of cost, DNA barcoding protocol is much more costly than the other two methods because it requires reagents for DNA sequencing, which are generally expensive. PCR-RFLP protocol requires reagents for PCR and restriction enzyme, which cost less than reagents required for sequencing. LAMP protocol needs reagents for LAMP, and also FD reagent if fluorescence method is used. The total cost of reagents used in LAMP and PCR-RFLP are variable depending on the reaction setting (e.g., how many restriction enzymes are used in PCR-RFLP; whether fluorescence method in LAMP is used or not). In the rough estimate, the cost of LAMP using fluorescence method would be almost twice the cost of PCR-RFLP protocol proposed for amphibians (e.g., Igawa et al., 2015), although it is still much lower than that of DNA barcoding.

Required reaction time in LAMP, $1-1.5 \mathrm{~h}$, is shorter than that of PCR-RFLP (usually $1-2 \mathrm{~h}$ for PCR and $1 \mathrm{~h}$ for enzyme digestion). In addition, LAMP assay can be performed in simple thermostat devices such as dry thermo unit, incubator, and heating bath, and does not necessarily require a thermal cycler because reaction condition of LAMP is essentially isothermal. This means the LAMP assay potentially can be conducted out of the laboratory: e.g., if a device which can keep temperature of reaction solution around $63-65^{\circ} \mathrm{C}$, the assay is executable even in the field. Although there are difficulties (e.g., avoiding contamination; stabilizing reaction system), it seems valuable to test the usability of LAMP in field studies.

In addition, LAMP assay has an advantage 
of flexibility in establishing experimental system. In theory, we can design the matched primer set to any sequences/SNPs representing taxon-specific difference in LAMP assay, whereas PCR-RFLP requires a precondition that the restriction enzyme matched to such sites exists.

These characteristics would be suitable not only for basic studies but also for conservation management. Accurate identification is the fundamental requirement in conservation activities, but this is not always easy. For example, sympatrically occurring pond frogs Pelophylax nigromaculatus and $P$. porosus are considered as threatened species by the Ministry of Environment of Japan (http:// www.jpnrdb.com/), but are hard to distinguish, especially in their early stages of growth. Thus, a quick identification method is required in the conservation activities for these species. With several advantages mentioned above, LAMP assay could be a useful tool for identifying morphologically similar species.

To confirm the accuracy of the assay in more detail, further study with applying it to more individuals from wider range is needed. Alignment of experimental condition is also a future task for making the assay more convenient.

\section{ACKNOWLEDGMENTS}

We thank Y. Misawa, T. Matsuki, and N. Yoshikawa for their help in sampling. We are also grateful to two anonymous reviewers for providing helpful comments that improved the early version of the manuscript. This work was supported by an internal grant from The Hakubi Center, Kyoto University to TH.

\section{LiterATURE Cited}

Ai, L., Li, C., Elsheikha, H. M., Hong, S. J., Chen, J. X., Chen, S. H., LI, X., CaI, X. Q., Chen, M. X., And Zhu, X. Q. 2010. Rapid identification and differentiation of Fasciola hepatica and Fasciola gigantica by a loopmediated isothermal amplification (LAMP) assay. Veterinary Parasitology 174: 228-233.

Aonuma, H., Suzuki, M., Iseki, H., Perera, N., Nelson, B., Igarashi, I., Yagi, T. Kanuka, H., AND Funumoto, S. 2008. Rapid identification of Plasmodium-carrying mosquitoes using loopmediated isothermal amplification. Biochemical and Biophysical Research Communications 376: 671-676.

Igawa, T., Kurabayashi, A., Nishioka, M., AND SumidA, M. 2006. Molecular phylogenetic relationship of toads distributed in the Far East and Europe inferred from the nucleotide sequences of mitochondrial DNA genes. Molecular Phylogenetics and Evolution 38: 250-260.

Igawa, T., Komaki, S., Takahara, T., and SumidA, M. 2015. Development and validation of PCR-RFLP assay to identify three Japanese brown frogs of the true frog genus Rana. Current Herpetology 34: 89-94.

Maeda, N. And Matsui, M. 1999. Frogs and Toads of Japan, Revised Edition. Bun-ichi Sogo Shuppan, Tokyo.

Matsui, M. 1976. A new toad from Japan. Contributions from the Biological Laboratory, Kyoto University 25: 1-10.

Matsui, M. 1984. Morphometric variation analyses and revision of the Japanese toads (Genus Bufo, Bufonidae). Contributions from the Biological Laboratory, Kyoto University 26: 209-428.

Mori, Y. AND Notami, T. 2009. Loop-mediated isothermal amplification (LAMP): A rapid, accurate, and cost-effective diagnostic method for infectious diseases. Journal of Infection and Chemotherapy 15: 62-69.

Notani, T., Okayama, H., Masubushi, H., YoneKawa, T., Watanabe, K., Amino, N., AND HASE, T. 2000. Loop-mediated isothermal amplification of DNA. Nucleic Acids Research 28: e63.

OKamoto, T. and Hikida, T. 2009. Three genetic lineages of the Japanese skink Plestiodon japonicus (Scincidae, Squamata) and the genetic composition of their contact zones. Journal of Zoological Systematics and Evolutionary Research 47: 181-188.

Accepted: 28 December 2015 